Support statement: This research was funded in part from a grant by Secretaria d'Universitats i Recerca del Departament d'Economia i Coneixement de la Generalitat de Catalunya, grant number GRC 2014 SGR 1569. Funding information for this article has been deposited with FundRef.

Conflict of interest: Disclosures can be found alongside the online version of this article at erj.ersjournals.com

\title{
References
}

1 Rochester D, Braun N. Determinants of maximal inspiratory pressure in chronic obstructive pulmonary disease. Am Rev Respir Dis 1985; 132: 42-47.

2 Ottenheijm CAC, Heunks LMA, Sieck GC, et al. Diaphragm dysfunction in chronic obstructive pulmonary disease. Am J Respir Crit Care Med 2005; 172: 200-205.

3 McKenzie DK, Butler JE, Gandevia SC. Respiratory muscle function and activation in chronic obstructive pulmonary disease. J Appl Physiol 2009; 107: 621-629.

4 Celli BR, MacNee W, Agusti A, et al. Standards for the diagnosis and treatment of patients with COPD: a summary of the ATS/ERS position paper. Eur Respir J 2004; 23: 932-946.

5 Finucane KE, Singh B. Human diaphragm efficiency estimated as power output relative to activation increases with hypercapnic hyperpnea. J Appl Physiol 2009; 107: 1397-1405.

6 Gosselink RA, Wagenaar RC, Rijswijk H, et al. Diaphragmatic breathing reduces efficiency of breathing in patients with chronic obstructive pulmonary disease. Am J Respir Crit Care Med 1995; 151: 1136-1142.

7 Similowski T, Yan S, Gauthier AP, et al. Contractile properties of the human diaphragm during chronic hyperinflation. N Engl J Med 1991; 325: 917-923.

8 Beck J, Sinderby C, Lindström L, et al. Effects of lung volume on diaphragm EMG signal strength during voluntary contractions. J Appl Physiol 1998; 85: 1123-1134.

9 Liu L, Liu H, Yang Y, et al. Neuroventilatory efficiency and extubation readiness in critically ill patients. Crit Care; 2012; 16: R143.

10 Petitjean M, Bellemare F. Phonomyogram of the diaphragm during unilateral and bilateral phrenic nerve stimulation and changes with fatigue. Muscle Nerve 1994; 17: 1201-1209.

11 Sarlabous L, Torres A, Fiz JA, et al. Index for estimation of muscle force from mechanomyography based on the Lempel-Ziv algorithm. J Electromyogr Kinesiol 2013; 23: 548-557.

12 Sarlabous L, Torres A, Fiz JA, et al. Evidence towards improved estimation of respiratory muscle effort from diaphragm mechanomyographic signals with cardiac vibration interference using sample entropy with fixed tolerance values. PLoS One 2014; 9: e88902.

13 Torres A, Fiz JA, Sarlabous L, et al. Noninvasive evaluation of the respiratory muscular function by means of mechanomyographic signals of the diaphragm muscle in COPD patients. Eur Respir J 2010; 36: Suppl. 54, 503s.

14 American Thoracic Society/European Respiratory Society: ATS/ERS statement on respiratory muscle testing. Am J Respir Crit Care Med 2002; 166: 518-624.

15 Bakke PS, Rönmark E, Eagan T, et al. Recommendations for epidemiological studies on COPD. Eur Respir J 2011; 38: 1261-1277.

16 Estrada L, Torres A, Sarlabous L, et al. Improvement in neural respiratory drive estimation from diaphragm electromyographic signals using fixed sample entropy. IEEE J Biomed Health Inform 2015 [in press; DOI: 10.1109/ JBHI.2015.2398934].

17 Duiverman ML, Van Eykern LA, Vennik PW, et al. Reproducibility and responsiveness of a noninvasive EMG technique of the respiratory muscles in COPD patients and in healthy subjects. J Appl Physiol 2004; 96: 1723-1729.

18 De Troyer A, Kirkwood PA, Wilson TA. Respiratory action of the intercostal muscles. Physiol Rev 2005; 85: $717-756$.

\section{Poor standardisation of plethysmographic specific airways resistance measurement despite widespread use}

To the Editor:

Plethysmographic specific airways resistance $\left(s R_{\mathrm{aw}}\right)$ has remained the most recognised measure of airway resistance for almost 60 years [1]. Studies in both adults and children (including preschoolers) suggest clinical utility across important obstructive lung diseases [2-5]. Measurement protocols based on rapid panting or tidal breathing exist, and are incorporated into many current commercial plethysmographs. Tidal breathing measurement offers feasibility across a wide age range, and a relatively stable index with 
which to distinguish effects of disease from those of growth and development [6]. Both adult and paediatric reference data exists $[7,8]$; however, the wide variation in methodology existing between centres has recently been highlighted [8], and $s R_{\mathrm{aw}}$ remains one of the few lung function measurements without formal standardisation guidelines [9]. Despite the wide availability of plethysmographic equipment in lung function laboratories, its use is frequently confined to lung volume measurements. Although simultaneous measures of airway resistance can easily be recorded at no extra cost, the clinical applications of $s R_{\mathrm{aw}}$ remain unclear. As part of ongoing $s R_{\mathrm{aw}}$ standardisation work, we sought to describe current international use of $s R_{\mathrm{aw}}$ across paediatric and adult respiratory laboratories.

Online surveys were distributed through members of four different societies: the European Respiratory Society, American Thoracic Society, Thoracic Society of Australia and New Zealand and the UK Association of Respiratory Technology and Physiology. Centres currently performing $s R_{\mathrm{aw}}$ testing were asked to complete the surveys and indicate whether measurements were performed for clinical and/or research purposes, the type of device used, age range tested and how many tests were performed each year. The questionnaire was intentionally brief to aid response rate. Respondents were asked to complete a second online questionnaire regarding specific methodology used (including respiratory rate targeted during measurements), clinical/ research situations of use, perceived clinical utility within their laboratory and $s R_{\mathrm{aw}}$ outcomes reported. $s R_{\mathrm{aw}}$ outcomes of interest were total resistance ( $\mathrm{s} R_{\mathrm{tot}}$; calculated as the difference between points of maximum plethysmographic box pressure), effective resistance ( $R_{\text {eff }}$, calculated from multiple points throughout the breathing cycle using an integration method), peak resistance (calculated between points of peak inspiratory and expiratory flow) and resistance over a fixed flow range $\left(\right.$ e.g. -0.5 to $\left.+0.5 \mathrm{~L} \cdot \mathrm{s}^{-1}\right)$ [8].

Overall, 47 centres indicated current use of $s R_{\mathrm{aw}}$, across 16 countries and four continents; the highest number of centres being in Europe (34 centres) and Australasia (10 centres). The greatest reported usage was within the Netherlands and UK (seven centres each), and Australia (six centres). Adults, school-aged, and pre-school children (aged <6 years) were assessed in $81 \%, 72 \%$ and $49 \%$ of centres, respectively. The majority of centres where adult testing was performed reported $>100$ adult tests per year, with $37 \%$ of centres reporting $>1000$ adult tests per year. The volume of tests performed in children was lower, with the majority of school age paediatric centres (55\%) performing 100-1000 tests per year whilst $74 \%$ of pre-school testing centres performed 10-100 tests per year.

A wide variety of commercial equipment existed across centres (eight different devices). Although most data had been collected using either Jaeger (27 centres) or SensorMedics (10 centres) equipment, both of which are now incorporated within CareFusion, several different software versions were being used within such devices. The remaining manufacturers were Medgraphics and Zan (three centres each), Medisoft (two centres), Morgan, Medical Equipment Europe and nSpire (one centre each).

Among the respondents completing the initial questionnaire, $87 \%$ indicated that $s R_{\mathrm{aw}}$ results were used clinically, while $57 \%$ used $s R_{\mathrm{aw}}$ in respiratory research and $36 \%$ were actively collecting healthy control data. This high reported clinical $s R_{\mathrm{aw}}$ use prompted distribution of the second more detailed questionnaire, which was completed by $77 \%$ of those initial respondents.

Significant variation of testing protocol was observed in the replies. The median (range) number of trials per session was 3 (1-5), with each trial comprising 5 (1-10) individual breaths. 14 centres reported a maximum
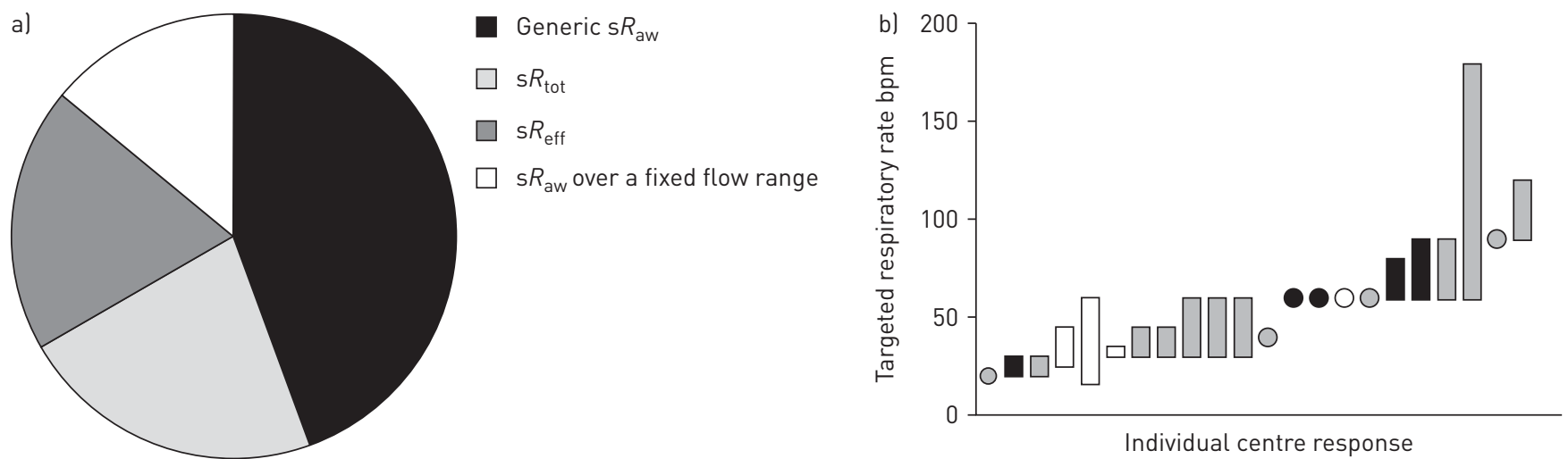

FIGURE 1 Summary of a) reported specific airways resistance ( $s R_{\text {aw }}$ ) outcomes (across 47 centres) and b) the targeted respiratory rates reported by the 22 centres (out of $36 ; 55 \%$ ) indicating this was part of local $s R_{\text {aw }}$ testing protocol. $s R_{\text {tot }}$ : total resistance; $s R_{\text {eff }}$ : effective resistance. b) Centres are categorised according to whether both paediatric and adult (grey), paediatric only (white), or adult only subjects are measured (black); centres indicating a specific target rate (breaths per minute; bpm) are shown as circular symbols and those indicating a respiratory rate range are shown as bars. 
number of attempted trials which ranged between 5 and 10 trials. Use of a specific target for breathing frequency during $s R_{\mathrm{aw}}$ measurements occurred at $72 \%$ of centres, but this target showed marked variation (figure 1). Only two centres varied the targeted rate according to the subject's age. The remaining $28 \%$ of centres specified either no target for respiratory rate (19\% of centres) or a "normal tidal breathing rate".

Reported parameters were poorly described by manufacturers, with almost half the centres (44\%) unable to give a full description of the primary outcome reported, simply indicating that the manufacturer reported a "generic $s R_{\mathrm{aw}}$ ". At the remaining centres, the primary outcome reported was $s R_{\text {tot }}(22 \%), \mathrm{s} R_{\mathrm{eff}}(19 \%)$, or $\mathrm{s} R_{\mathrm{aw}}$ over a fixed flow range (14\%). Five centres (14\%) indicated use of more than one outcome, depending on the clinical situation. Among the $72 \%$ of centres where relevant information concerning equipment software was retrievable, results were summarised as median values in $35 \%$ and mean in the remaining $65 \%$. One centre reported results from only one acceptable trial. Despite recent recommendations [8], manual adjustment of automatically generated tangents of pressure-flow loops was still occurring at $42 \%$ of centres. Abnormal results were defined based on reference equations at over half the centres (58\%), but the majority (12/21) could not identify which equation was used in the equipment. The other nine centres used several different equations, including those of KirKBY et al. [8] (2010), BRISCOE and DubOIs [10] (1958), and in-house reference equations based on self-collected data. A fixed "upper limit of normal", set as default by the manufacturer without specification of reference material, was used by the remaining $42 \%$ of centres.

Consistent with responses from the first questionnaire, 30 respondents (83\%) to the second questionnaire indicated clinical use of $s R_{\mathrm{aw}}$. All performed baseline measurements, with $61 \%$ also using $s R_{\mathrm{aw}}$ to assess bronchodilator response and $23 \%$ for bronchial challenge (four out of seven of whom indicated a change in standard testing protocol when $s R_{\mathrm{aw}}$ was used for such purposes). All 30 centres stated that they found $\mathrm{s} R_{\mathrm{aw}}$ measurements clinically useful across a wide range of respiratory conditions, but primarily in the evaluation of obstructive lung disease. Utility from $s R_{\mathrm{aw}}$ was perceived for asthma (all 13 centres providing more detailed information), cystic fibrosis, chronic obstructive pulmonary disease, interstitial lung disease and bronchopulmonary dysplasia. Utility was perceived to be higher when other lung function techniques, such as spirometry, were not technically feasible for the patient.

These results highlight widespread paediatric and adult use of $s R_{\mathrm{aw}}$ in respiratory function laboratories, centred in Europe and Australasia. Popularity probably reflects relative ease of data collection and availability of suitable equipment, but also high perceived clinical utility (almost $90 \%$ of respondents). This occurred despite striking lack of agreement with respect to methodology, outcomes and reference data. Variation in several of these important methodological aspects can significantly affect recorded $s R_{\mathrm{aw}}$ values $[8,11]$ and interpretation in the clinical setting [12]. Publication of recommendations in 2010 for testing protocol using the most common device in our survey has failed to prevent marked variation in practice [8]. These findings and the high reported volume of current testing highlight the urgent need for generalisable recommendations to standardise all aspects of $s R_{\mathrm{aw}}$ measurement and interpretation.

The formation of a task force, endorsed by international respiratory societies, and including representation from countries with heavy current use, would be an important step towards optimising potential clinical utility of this widely used technique. Until standardisation has been achieved, these authors would advise centres to adhere to existing recommendations [8] and interpret $s R_{\mathrm{aw}}$ results with caution.

@ERSpublications

sRaw is widely used but results should be interpreted with caution until its recording is better standardised http://ow.ly/QvJRJ

Paul D. Robinson ${ }^{1,2}$, Janet Stocks ${ }^{2}$, Francois Marchal ${ }^{3}$, Kim G. Nielsen ${ }^{4}$, Bruce R. Thompson ${ }^{5}$, Waldemar Tomalak ${ }^{6}$ and Jane Kirkby ${ }^{2}$

${ }^{1}$ Children's Hospital at Westmead, Sydney, Australia. ${ }^{2}$ University College London, Institute of Child Health, London, UK. ${ }^{3}$ Hopital d'Enfants, Explorations Fonctionnelles Pédiatriques, Vandoeuvre, France. ${ }^{4}$ Paediatric Pulmonary Service, Rigshospitalet, Copenhagen, Denmark. ${ }^{5}$ Alfred Hospital, Melbourne, Australia. ${ }^{6}$ National Research Institute for Tuberculosis and Lung Diseases, Rabka Branch, Rabka, Poland.

Correspondence: Paul D. Robinson, Dept of Pediatric Respiratory Medicine, The Children's Hospital at Westmead, Locked Bag 4001, Westmead, Sydney, NSW 2145, Australia. E-mail: paul.robinson1@health.nsw.gov.au

Received: Oct 112014 | Accepted after revision: June 192015 | First published online: Aug 202015

Conflict of interest: None declared.

References

1 Dubois AB, Brody AW, Lewis DH, et al. Oscillation mechanics of lungs and chest in man. J Appl Physiol 1956; 8: 587-594.

2 Nielsen KG, Bisgaard H. Cold air challenge and specific airway resistance in preschool children. Paediatr Respir Rev 2005; 6: 255-266. 
3 Nielsen KG, Pressler T, Klug B, et al. Serial lung function and responsiveness in cystic fibrosis during early childhood. Am J Respir Crit Care Med 2004; 169: 1209-1216.

4 Klug B, Bisgaard H. Measurement of lung function in awake 2-4-year-old asthmatic children during methacholine challenge and acute asthma: a comparison of the impulse oscillation technique, the interrupter technique, and transcutaneous measurement of oxygen versus whole-body plethysmography. Pediatr Pulmonol 1996; 21: 290-300.

5 Van Noord JA, Smeets J, Clement J, et al. Assessment of reversibility of airflow obstruction. Am J Respir Crit Care Med 1994; 150: 551-554.

Nielsen KG. Plethysmographic specific airway resistance. Paediatr Respir Rev 2006; 7: Suppl 1, S17-S19.

7 Piatti G, Fasano V, Cantarella G, et al. Body plethysmographic study of specific airway resistance in a sample of healthy adults. Respirology 2012; 17: 976-983.

8 Kirkby J, Stanojevic S, Welsh L, et al. Reference equations for specific airway resistance in children: the asthma UK initiative. Eur Respir J 2010; 36: 622-629.

9 Rosenfeld M, Allen J, Arets BH, et al. An official American Thoracic Society workshop report: optimal lung function tests for monitoring cystic fibrosis, bronchopulmonary dysplasia, and recurrent wheezing in children less than 6 years of age. Ann Am Thorac Soc 2013; 10: S1-S11.

10 Briscoe WA, Dubois AB. The relationship between airway resistance, airway conductance and lung volume in subjects of different age and body size. J Clin Invest 1958; 37: 1279-1285.

11 Coutier L, Varechova S, Demoulin B, et al. Specific airway resistance in children: panting or tidal breathing? Pediatr Pulmonol 2014; 49: 245-251.

12 Coutier L, Ioan I, Sadegh-Eghbali A, et al. Flow dependence of specific airway resistance and diagnostic of asthma in children. Pediatr Pulmonol 2015; 50: 1107-1112.

\section{Multiple breath washouts in children can be shortened without compromising quality}

To the Editor:

Lung clearance index (LCI) derived from multiple breath washout (MBW) is a sensitive, noninvasive measure of ventilation heterogeneity and is used for the assessment of cystic fibrosis (CF) [1], asthma [1] and primary ciliary dyskinesia (PCD) [2]. LCI is the number of lung turnovers (functional residual capacity (FRC)) required to washout an inhaled inert gas to $1 / 40$ of its initial concentration: a historically set end-point of no physiological significance [3]. The number of lung turnovers needed to wash out the gas, and hence the LCI, increases with disease severity. It is non-effort-dependent and requires only passive cooperation. The test can be time-consuming, especially with severe airway obstruction as more time is required to wash out the tracer gas. This is a particular problem in young children, in whom a test lasting several minutes is intolerable. This could be shortened by stopping the test at a higher concentration of tracer gas. We propose that instead of $1 /$ 40 of the initial concentration being used as an end-point ("LCI standard" (LCIstd)), the concentration could be raised to $1 / 30$ (LCI0.75), 1/20 (LCI0.5) or 1/10 (LCI0.25) of the starting concentration of tracer gas, or using a fixed time period of washout, for example $20 \mathrm{~s}$, or a fixed number of breaths.

In 17 preschool children (median age 3.9 years), our pilot data showed that only $35 \%$ could perform LCIstd, but 65\% completed LCI0.5 [4].

Two studies, demonstrated that LCI0.5 can be performed in school age children with CF with the same diagnostic performance as LCIstd [5, 6] and a recent study [7] showed LCI0.5 is sensitive to improvements with dornase alpha and hypertonic saline in CF. We aimed to investigate the utility of shortened washouts in school age children with asthma, PCD and CF, and to assess the sensitivity of change in LCI to two interventions (intravenous antibiotics in $\mathrm{CF}$ [8] and intramuscular triamcinolone injection in severe asthma [9]). We hypothesised that LCI measurements can be shortened without compromising the quality of the information obtained and are as responsive to therapeutic intervention as LCIstd.

We performed a retrospective analysis of previously collected data from MBW measurements at the Royal Brompton Hospital (London, UK) between January 2008 and May 2014. All research studies had been approved by the appropriate research ethics committees, and informed consent obtained. Data from 20 children with CF (median age 13.85 years, seven male), 19 with PCD (median age 13.89 years, six male), 21 with asthma (median age 13.29 years, 12 male) and 17 healthy controls (median age 9.76 years, nine male) were analysed initially, followed by data from 32 children with asthma who had MBW prior to and 1 month after an intramuscular injection of triamcinolone, and a cohort of 17 people with CF who had MBW at the beginning and end of a course of intravenous antibiotics. LCIstd data for the CF intervention cohort have been reported 